6. Lewis BB, Buffie CG, Carter RA, et al. Loss of microbiota-mediated colonization resistance to Clostridium difficile infection with oral vancomycin compared to metronidazole. J Infect Dis 2015;212:1656-1665.

7. Barlett JG. The case for vancomycin as the preferred drug for treatment of Clostridium difficile infection. Clin Infect Dis 2008;46:1489-1492.

8. Pepin J. Vancomycin for the treatment of Clostridium difficile infection: for whom is this expense bullet really magic? Clin Infect Dis 2008:46: $1493-1498$.
9. Abujamel T, Cadnum JL, Jury LA, et al. Defining the vulnerable period for re-establishment of Clostridium difficile colonization after treatment of $C$. difficile infection with oral vancomycin or metronidazole. PLoS One 2013;8(10):e76269.

10. Oral vancomycin to prevent recurrent Clostridium difficile infection with antibiotics. ClinicalTrials.gov Identifier: NCT03466502.

11. Efficacy of oral vancomycin prophylaxis for prevention of recurrent Clostridium difficile infection. ClinicalTrials.gov Identifier: NCT03462459.

\title{
Increased time spent on terminal cleaning of patient rooms may not improve disinfection of high-touch surfaces
}

\author{
Edmond A. Hooker MD, DrPH ${ }^{1,2}$ (1] \\ Xavier University, Cincinnati, Ohio and University of Cincinnati, Cincinnati, Ohio
}

To the Editor - I read with great interest the study by Coppin et al, ${ }^{1}$ in which they attempted to determine whether increased cleaning time would improve disinfection of high-touch surfaces. ${ }^{1}$ Although I applaud the attempt to address the issue, I am concerned about the very limited description of their methods. I also feel that the methodological flaws limit any conclusions from their research.

First, the authors allowed for the use of 3 very different disinfectants. They did not describe how each was utilized. Also, they did not describe what the EVS staff did for the increased time. If the environmental services staff (EVS) was not cleaning first and then disinfecting, it is not surprising that there was limited effect. Doing something wrong for longer does not improve cleaning. Also, previous research that showed EVS was much less effective at disinfection when they were not monitored. ${ }^{2}$ They only cultured 5 surfaces, and they did not culture the mattress, which is the highest touch point for the patient.

The bed manufacturers, in their revised instructions for use, require 5-6 steps to be performed when terminally cleaning a bed. These steps, if done properly, require 35 minutes to 1 hour to complete.

The authors also did not report how they cultured for bacteria. This could have made a huge difference in the results of the study. There is no description of the actual colony-forming units of bacteria found in each group before and after cleaning. The authors should have reported the log reduction in bacteria for each group, and statistical significance should have been reported as well.
The authors instead report predicted counts, which makes interpretation almost impossible.

I am concerned that this research will send the wrong message to hospitals: indicating that better cleaning will not work. It is extremely concerning that this research was funded by a manufacturer of an ultraviolet light disinfection machine. I strongly believe that cleaning better will not only decrease bacterial counts, but it will also decrease infections.

Author ORCIDs. Edmond A. Hooker, (D) https://orcid.org/0000-0001-73079483

Financial support. No financial support was provided relevant to this article.

Conflicts of interest. All authors report no conflicts of interest relevant to this article.

\section{References}

1. Coppin JD, Villamaria FC, Williams MD, Copeland LA, Zeber JE, Jinadatha C. Increased time spent on terminal cleaning of patient rooms may not improve disinfection of high-touch surfaces. Infect Control Hosp Epidemiol 2019:1-2.

2. Korchinski K, Hinkle C, Sopirala M. Effect of automated ultraviolet-C emitting device on disinfection of hospital rooms with and without real-time auditing of cleaning process. In: Open Forum Infectious Diseases, Vol. 3. New York: Oxford University Press; 2016.
Author for correspondence: Edmond Hooker, MD, DrPH, 3800 Victory Parkway, ML 5141, Cincinnati, OH 45207-5141. E-mail: hookere@xavier.edu

Cite this article: Hooker EA. (2019). Increased time spent on terminal cleaning of patient rooms may not improve disinfection of high-touch surfaces. Infection Control \& Hospital Epidemiology, 40: 1086, https://doi.org/10.1017/ice.2019.181 